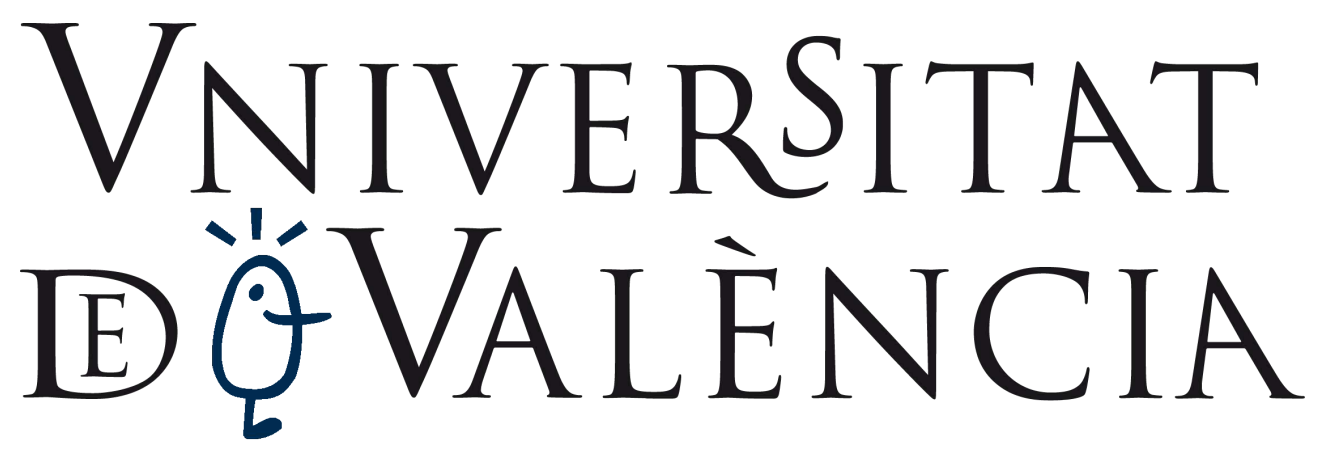

Citation for the original published paper:

Jing-ya LI, Xiao-dong XU, Hui ZHANG, Xiao-feng TAO, Svensson Tommy, Botella Carmen, Xin CHEN, Multi-beam cooperative frequency reuse for coordinated multi-point transmission,

The Journal of China Universities of Posts and Telecommunications,

Volume 17, Issue 6, 2010, Pages 11-18, ISSN 1005-8885

https://doi.org/10.1016/S1005-8885(09)60519-7

The published paper can be obtained in the following link:

https://www.sciencedirect.com/science/article/pii/S1005888509605197

This is an author's version under creative common license CC-BY-NC-ND 4.0 


\title{
Multi-beam cooperative frequency reuse for coordinated multi-point transmission
}

\author{
LI Jing-ya ${ }^{1}(\bowtie)$, XU Xiao-dong ${ }^{1}$, ZHANG Hui ${ }^{1}$, TAO Xiao-feng ${ }^{1}$, \\ Tommy Svensson ${ }^{2}$, Carmen Botella ${ }^{2}$, CHEN Xin ${ }^{1}$ \\ 1. Wireless Technology Innovation Institute, Key Laboratory of Universal Wireless Communications, Ministry of Education, \\ Beijing University of Posts and Telecommunications, Beijing 100876, China \\ 2. Signals and Systems, Chalmers University of Technology, SE-412-96 Gothenburg, Sweden
}

\begin{abstract}
Coordinated multi-point (CoMP) joint transmission is considered in the 3rd generation partnership project (3GPP) long term evolution (LTE)-advanced as a key technique to mitigate inter-cell interference and improve the cell-edge performance. To effectively apply CoMP joint transmission, efficient frequency reuse schemes need to be designed to support resource management cooperation among coordinated cells. However, most of the existing frequency reuse schemes are not suitable for CoMP systems due to not considering multi-point joint transmission scenarios in their frequency reuse rules. In addition, the restrictions of frequency resources in those schemes result in a high blocking probability. To solve the above two problems, a multi-beam cooperative frequency reuse (MBCFR) scheme is proposed in this paper, which reuses all the available frequency resources in each sector and supports multi-beam joint transmission for cell-edge users. Besides, the blocking probability is proved to be efficiently reduced. Moreover, a frequency-segment-sequence based MBCFR scheme is introduced to further reduce the inter-cell interference. System level simulations demonstrate that the proposed scheme results in higher cell-edge average throughput and cell-average throughput with lower blocking probability.
\end{abstract}

Keywords frequency reuse, inter-cell interference, coordinated multi-point transmission, LTE-advanced

\section{Introduction}

Orthogonal frequency division multiplexing (OFDM) based multiple access is considered as a promising radio access technology for the 4th generation (4G) mobile communication systems. Orthogonal frequency division multiple access (OFDMA) has been adopted as the downlink access technology of the 3GPP LTE and LTE-advanced [1-2]. Based on the OFDM technique, OFDMA inherits the immunity to intra-cell interference. However, the inter-cell interference (ICI) is still a major issue [3]. In fact, frequency reuse factor being equal to one causes serious ICI to users in the cell-edge areas, leading to poor cell-edge throughput.

CoMP joint transmission was proposed in 3GPP LTE-advanced as a key technique to mitigate ICI and further

Corresponding author: LI Jing-ya, E-mail: lijingya1986@gmail.com improve the cell-edge performance [4]. In this approach, if both data and channel of all users could be shared in real time, adjacent base stations could act as a single and distributed antenna array and hence, data to a user is simultaneously transmitted from multiple base stations to improve the received signal quality. Notice that this technique was formerly referred as network coordination [5].

To effectively apply CoMP joint transmission, resource management cooperation among coordinated cells is necessary. One promising technique for multi-cell joint resource management is frequency reuse, which restricts the available frequency resources of different cells through a predefined frequency reuse rule, reducing the signaling overhead required for inter-cell information exchange and the amount of feedback needed from the users. The most common frequency reuse schemes are soft frequency reuse (SFR) and fractional frequency reuse (FFR) [6-9]. These schemes are based on the idea of applying a frequency reuse factor of one 
in the cell-center areas, and using different frequency segments in the cell-edge areas of neighboring cells. In Ref. [10], a frequency reuse scheme is proposed for a switched-beam system with three-sector cells, where orthogonal frequency resources are allocated to different sectors of each cell and users are served with the appropriate set of subcarriers according to their assigned beam. Based on Ref. [10], the use of different subcarriers for cell-edge users and cell-center users is considered to further improve the system throughput in Ref. [11]. However, since only one third of subcarriers are available for each sector, the resulting blocking probability is high in these two schemes. In addition, all of the above schemes use different frequency segments in the cell-edge areas of neighboring cells, without considering a multi-point joint transmission scenario in their frequency reuse rules. Hence, these schemes are not suitable for CoMP systems.

In this paper, a novel frequency reuse scheme, named MBCFR, is proposed for CoMP systems to support multi-point joint transmission. In this scheme, all the available frequency resources can be reused in each sector, and neighboring beams belonging to different cells are assigned with the same frequency resources so that multi-beam joint transmission can be supported. Besides, the blocking probability is proved to be efficiently reduced. Furthermore, in order to further reduce the number of interference beams due to co-channel interference, a frequency-segment-sequence based MBCFR (FSS-MBCFR) scheme is proposed, where the major ICI can be effectively avoided when the traffic load is light. Compared with the frequency reuse schemes in Refs. [10-11], simulation results demonstrate that the proposed FSS-MBCFR scheme results in higher cell-edge average throughput and cell-average throughput with lower blocking probability.

The rest of this paper is organized as follows. In Sect. 2, the system model for downlink multi-beam joint transmission is described. In Sect. 3, the MBCFR scheme is proposed for multi-beam joint transmission. Then, the FSS-MBCFR scheme is introduced to further reduce the ICI in Sect. 4. System level simulation results are provided in Sect. 5. Finally, Sect. 6 concludes the paper.

\section{System model}

A downlink OFDMA switched-beam system for multi-beam joint transmission is described in Fig. 1. In the system, each cell consists of three sectors, with each sector covered by two beams marked with Beam 1 (the white beams in Fig. 1) and Beam 2 (the zebra beams in Fig. 1), respectively.

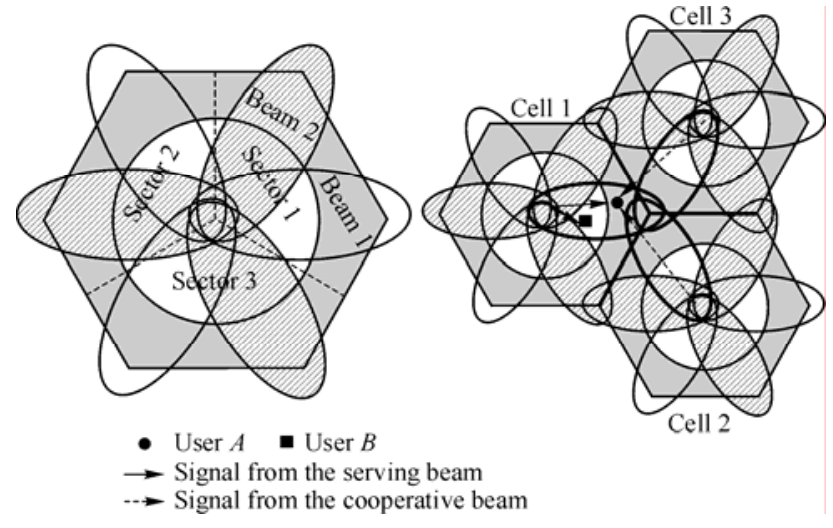

Fig. 1 System model for downlink multi-beam joint transmission

Users in each sector are divided into two groups according to the long term gain between the user and its serving base station. The long term gain consists of the path loss and the shadow fading. When the long term gain is higher than a pre-defined threshold, the user is treated as a cell-center user (CCU), e.g., user $B$ in Fig. 1. Each CCU can only communicate with its serving beam, which is the highest-power beam in the sector that the user belongs to. When the long term gain is less than a pre-defined threshold, the user is regarded as a cell-edge user (CEU), e.g., user $A$ in Fig. 1. Each CEU has a CoMP cooperating beam-set (CCBS), which is formed by the beams that provide data transmission service to this CEU, including its own serving beam. Hence, each CEU can be simultaneously served by the beams contained in its CCBS with the same frequency resources. In this paper, the CCBSs are pre-defined considering the three neighboring beams belonging to different cells. The different CCBSs are disjoint.

In the example of Fig. 1, the CCBS of the CEU A is formed by the three adjacent beams marked with Beam 1 in three neighboring sectors (Sector 1 of Cell 1, Sector 2 of Cell 2, Sector 3 of Cell 3 ). Hence, user $A$ can be simultaneously served by these three beams within the same frequency resources. In this example, user $B$ is a CCU and it can only communicate with its serving beam, i.e., Beam 1 in Sector 1 of Cell 1.

\subsection{Beamforming model}

The beamforming model considered in this system is the conventional beamformer for a uniform linear antenna array. 
According to Ref. [12], the array factor for its power radiation pattern is given as:

$\omega_{\mathrm{AF}}(\theta)=\frac{1}{m}\left|\frac{\sin \left\{\frac{m \pi}{2}(\cos \theta-\cos \phi)\right\}}{\sin \left\{\frac{\pi}{2}(\cos \theta-\cos \phi)\right\}}\right|^{2}$

where $m$ is the number of transmit antennas, $\phi$ is the boresight direction towards which the beam is steered and $\theta$ is the angle that the mobile forms with the antenna-array axis.

The gain of a single antenna is given based on Ref. [1]:

$\omega_{\mathrm{AG}}(\varphi)=\omega_{\mathrm{AG}}^{\max }-\min \left\{12\left(\frac{\varphi}{\varphi_{3 \mathrm{~dB}}}\right)^{2}, \omega_{\mathrm{AG}}^{\mathrm{FB}}\right\}$

where $\omega_{\mathrm{AG}}^{\max }$ is the maximum antenna gain in the boresight direction, $\varphi$ is the angle that the user forms with the sector boresight, $\varphi_{3 \mathrm{~dB}}$ is the angle associated with half power beamwidth and $\omega_{\mathrm{AG}}^{\mathrm{FB}}$ is the front to back power ratio.

\subsection{Interference and channel capacity}

The basic resource element considered in the system is the frequency resource block (RB), which consists of twelve contiguous subcarriers. It is assumed that each RB can only be assigned to one user. Let $\Psi_{k}$ denote the CCBS of a CEU $k$. Define $\bar{\Psi}_{k}$ to be the complement set of $\Psi_{k}$. Then, the signal to interference plus noise ratio (SINR) on RB $l$ for the active CEU $k$ connected to sector $j$ is determined as follows:

$\gamma_{j}^{k, l}=\frac{\sum_{s \in \Psi_{k}} P_{s, l} G_{s}^{k}\left|h_{s, l}^{k}\right|^{2} a_{s}^{k}}{\sum_{n \in \Psi_{k}} x_{n, l} P_{n, l} G_{n}^{k} a_{s}^{k}+N_{0}}$

where $P_{s, l}$ is the transmission power from the beam $s$ on RB l. $G_{s}^{k}$ and $a_{s}^{k}$ denote, respectively, the long term gain and the power radiation pattern between CEU $k$ and the sector that beam $s$ belongs to. $h_{s, l}^{k}$ denotes the fast fading gain on RB $l$

for the channel between CEU $k$ and the sector that beam $s$ belongs to. $N_{0}$ is the noise power received within each RB. Finally, $x_{n, l}$ is the allocation indicator of the $l$ th $\mathrm{RB}$, which can be given by:

$x_{n, l}= \begin{cases}1 ; & \mathrm{RB} l \text { is used in beam } n \\ 0 ; & \text { otherwise }\end{cases}$

In Ref. [1], it was pointed out that interference coordination is handled by the system once every $100 \mathrm{~ms}$. The information reported by the users and used by the system is the average SINR value. Thus, $\left|h_{s, l}^{k}\right|^{2}$ is replaced by its mean value. We assume that $E\left(\left|h_{s, l}^{k}\right|^{2}\right)=1$. Then, Eq. (3) can be expressed as: $\gamma_{j}^{k, l}=\frac{\sum_{s \in \Psi_{k}} P_{s, l} G_{s}^{k} a_{s}^{k}}{\sum_{n \in \bar{\Psi}_{k}} x_{n, l} P_{n, l} G_{n}^{k} a_{s}^{k}+N_{0}}$

In the case of the CCUs, they are only served by their serving beams. The average SINR expression on RB $l$ for $\mathrm{CCU} k$ of sector $j$ is then given by:

$\gamma_{j}^{k, l}=\frac{P_{j, l} G_{j}^{k} a_{j}^{k}}{\sum_{n \neq j} x_{n, l} P_{n, l} G_{n}^{k} a_{s}^{k}+N_{0}}$

Finally, according to Shannon theorem, the corresponding capacity to the user average SINR on RB $l$ can be expressed as:

$C_{j, l}^{k}=B \mathrm{lb}\left(1+\frac{\gamma_{j, l}^{k}}{\Gamma}\right)$

where $B$ is the bandwidth of each RB, and $\Gamma$ is the SINR gap, which is a constant related to the target bit error rate (BER) given as [13]:

$\Gamma=\frac{-\ln \left(5 R_{\mathrm{BER}}\right)}{1.5}$

where $R_{\mathrm{BER}}$ is the $\mathrm{BER}$.

\section{Multi-beam cooperative frequency reuse}

In this section, we introduce the MBCFR scheme for OFDMA switched-beam systems, which can support multi-beam joint transmission for CEUs and significantly reduce the blocking probability.

\subsection{Frequency reuse rule}

In order to reduce the blocking probability as well as support multi-beam joint transmission for CEUs, the resources are assigned to each sector according to the following frequency reuse rule:

Step 1 The whole frequency resources, denoted as $F$, are divided into two orthogonal sets and respectively marked with $F_{1}$ and $F_{2}$.

Step $2 \quad F_{1}$ is assigned to the beams marked with Beam 1, and $F_{2}$ is assigned to the beams marked with Beam 2 . Hence, Beam 1 and Beam 2 will occupy different frequency resources.

Step 3 The available frequency resources of both beams are further divided into two orthogonal subsets, denoted as $F_{i}^{\mathrm{E}}$ and $F_{i}^{\mathrm{C}}$, respectively, where $i \in\{1,2\}$. 
Step $4 F_{i}^{\mathrm{E}}$ is assigned for CEUs in the CCBS to which Beam $i$ belongs, and $F_{i}^{\mathrm{C}}$ is assigned for CCUs in Beam $i$.

Based on the proposed frequency reuse rule, the frequency resources assignment for each cell in the MBCFR scheme is shown in Fig. 2. On the one hand, the neighboring beams in each cell are assigned with different frequency sets $F_{1}$ and $F_{2}$. Therefore, the intra-cell interference can be effectively mitigated. On the other hand, as shown in Fig. 2(b), the adjacent beams in each CCBS reuse the same subcarriers in cell-edge areas and multi-beam joint transmission can be supported. By this means, the two major interference beams of each CEU are contained in its CCBS. Hence, these two beams will provide data transmission service to the CEU instead of introducing interference signals and the CEUs' performance can be further improved.

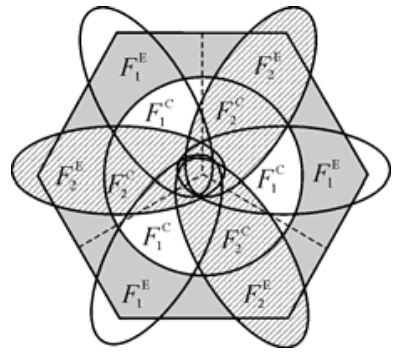

(a) Frequency reuse rule for each cel

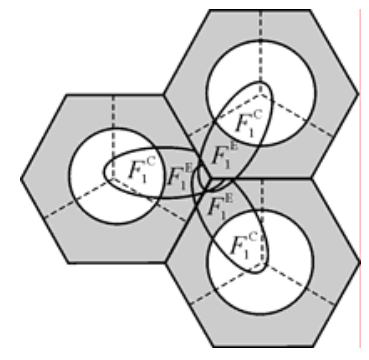

(b) Frequency assignment for one CCBS
Fig. 2 Frequency resources assignment in the MBCFR scheme

Fig. 3 shows the schemes proposed in Refs. [10-11], named as single-beam frequency reuse 1 (SBFR-1) and single-beam frequency reuse 2 (SBFR-2), respectively. In SBFR-1, the available frequency resources are split into three sets, one for each sector. Then, the frequency resources of each sector are further divided into two subsets, one for each beam. For example, as shown in Fig. 3(a), $f_{1}$ and $f_{2}$ belong to the frequency set assigned to Sector $1, f_{1}$ for Beam 1 and $f_{2}$ for Beam 2. In SBFR-2, the frequency resources for each beam are further divided into two parts for CEUs and CCUs, respectively (see Fig. 3(b)).

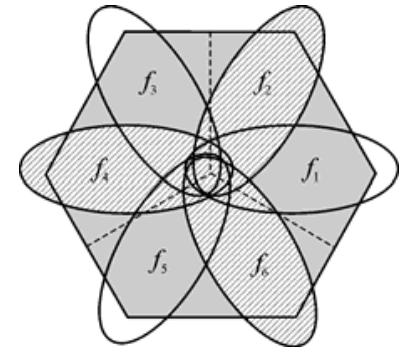

(a) SBFR-1

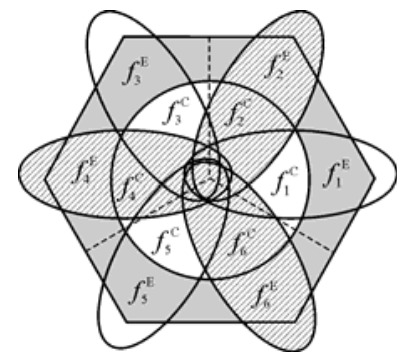

(b) SBFR-2
Fig. 3 Frequency resources assignment in Refs. [10-11], respectively
We can see that in Refs. [10-11], different sectors of each cell are allocated with orthogonal frequency resources, so the available frequency resources for adjacent beams in each CCBS are different. Hence, different from our proposed MBCFR scheme, multi-beam joint transmission cannot be supported in the SBFR-1 and SBFR-2 schemes.

In addition, in our proposed MBCFR scheme, the whole frequency resources are reused in all sectors with frequency reuse factor of one. However, in the SBFR-1 and SBFR-2 schemes, only one third of the frequency resources are available for each sector. Hence, the number of available frequency resources for each beam in the MBCFR scheme increases. In other words, the blocking probability due to the lack of frequency resources can be effectively reduced. In the next subsection, the blocking probability of the MBCFR scheme is studied and compared with the blocking probability of the schemes in Refs. [10-11].

\subsection{Blocking probability}

For each sector, let $n_{i}$ denote the number of users that need to be served in Beam $i$, where $n_{i}^{\mathrm{C}}$ and $n_{i}^{\mathrm{E}}$ denote the number of CCUs and CEUs of Beam $i$, respectively. Note that $n_{i}^{\mathrm{C}}+n_{i}^{\mathrm{E}}=n_{i} \cdot|A|$ denotes the number of RBs in set $A$. We assume that only one RB can be assigned to each active user.

In the proposed MBCFR scheme, users are divided into two groups and the blocking probability of CCUs and CEUs is independent. The cell-center frequency allocation is made in each sector in a centralized way, which means that each $\mathrm{CCU}$ only communicates with its serving beam and occupies one RB. Hence, the maximum number of accepted CCUs in Beam $i$ is $\left|F_{i}^{\mathrm{C}}\right|$.

Then, the cell-center blocking probability per sector due to the lack of frequency resources can be expressed by:

$P_{\mathrm{b} \_\mathrm{MBCFR}}^{\mathrm{C}}=\frac{\left[n_{1}^{\mathrm{C}}-F_{1}^{\mathrm{C}}\right]^{+}+\left[n_{2}^{\mathrm{C}}-F_{2}^{\mathrm{C}}\right]^{+}}{n_{1}+n_{2}}$

where $[a]^{+}=\left\{\begin{array}{ll}a ; & a \geqslant 0 \\ 0 ; & a=0\end{array}\right.$.

The cell-edge frequency allocation of the proposed MBCFR scheme is made in a coordinated way in each CCBS, so each CEU is simultaneously served with the same RB by the three beams marked with Beam $i$ contained in its CCBS. Hence, each CEU occupies three RBs, one in each beam. The maximum number of accepted CEUs in the CCBS is $\left|F_{i}^{\mathrm{E}}\right|$. Since each CCBS consists of three beams, the cell-edge blocking probability per sector can be calculated by: 
$P_{\mathrm{b}_{-} \mathrm{MBCFR}}^{\mathrm{E}}=\frac{\left[n_{1}^{\mathrm{E}}-\frac{\left|F_{1}^{\mathrm{E}}\right|}{3}\right]^{+}+\left[n_{2}^{\mathrm{E}}-\frac{\left|F_{2}^{\mathrm{E}}\right|}{3}\right]^{+}}{n_{1}+n_{2}}$

Finally, from Eqs. (9) and (10), the blocking probability per sector of the proposed MBCFR scheme can be derived as:

$$
\begin{aligned}
P_{\mathrm{b}}^{\mathrm{MBCFR}}= & P_{\mathrm{b} \_ \text {MBCFR }}^{\mathrm{C}}+P_{\mathrm{b}_{\mathrm{B}} \mathrm{EBCFR}}^{\mathrm{E}}=\left\{\left[n_{1}^{\mathrm{C}}-F_{1}^{\mathrm{C}}\right]^{+}+\left[n_{2}^{\mathrm{C}}-F_{2}^{\mathrm{C}}\right]^{+}+\right. \\
& {\left.\left[n_{1}^{\mathrm{E}}-\frac{\left|F_{1}^{\mathrm{E}}\right|}{3}\right]^{+}+\left[n_{2}^{\mathrm{E}}-\frac{\left|F_{2}^{\mathrm{E}}\right|}{3}\right]\right\}\left(n_{1}+n_{2}\right)^{-1} }
\end{aligned}
$$

In the two reference schemes (the SBFR-1 scheme and the SBFR-2 scheme), only one third of frequency resources are available for each sector, and each user occupies one RB. In the SBFR-1 scheme, the RBs for each sector are randomly assigned to the different users irrespective of their category. Hence, the blocking probability of the SBFR-1 scheme can be expressed by:

$P_{\mathrm{b}}^{\text {SBFR_1 }}=\frac{\left[n_{1}-\frac{\left|F_{1}\right|}{3}\right]^{+}+\left[n_{2}-\frac{\left|F_{2}\right|}{3}\right]^{+}}{n_{1}+n_{2}}$

Similarly, the blocking probability of the SBFR-2 can be expressed by:

$P_{\mathrm{b}}^{\text {SBFR } 2}=\frac{\left[n_{1}^{\mathrm{C}}-\frac{F_{1}^{\mathrm{C}}}{3}\right]^{+}+\left[n_{2}^{\mathrm{C}}-\frac{F_{2}^{\mathrm{C}}}{3}\right]^{+}+\left[n_{1}^{\mathrm{E}}-\frac{\left|F_{1}^{\mathrm{E}}\right|}{3}\right]^{+}+\left[n_{2}^{\mathrm{E}}-\frac{\left|F_{2}^{\mathrm{E}}\right|}{3}\right]^{+}}{n_{1}+n_{2}}$

From Eqs. (11) and (13), it is obvious that $P_{\mathrm{b}}^{\mathrm{MBCFR}} \leqslant P_{\mathrm{b}}^{\text {SBFR } \_2}$. Since $[a+b]^{+} \leqslant[a]^{+}+[b]^{+}$, according to Eqs. (12) and (13), we have $P_{\mathrm{b}}^{\mathrm{SBFR} \_1} \leqslant P_{\mathrm{b}}^{\mathrm{SBFR} \_2}$. Let $\left|F_{i}^{\mathrm{C}}\right| /\left|F_{i}^{\mathrm{E}}\right|=n_{i}^{\mathrm{C}} / n_{i}^{\mathrm{E}}$, which means that the frequency subsets of each beam are divided according to the proportion of the number of CCUs and CEUs. Then, according to Eqs. (11) and (12), we can get $P_{\mathrm{b}}^{\mathrm{MBCFR}}(n) \leqslant P_{\mathrm{b}}^{\mathrm{SBFR}-1}(n)$. Hence, $P_{\mathrm{b}}^{\mathrm{MBCFR}}(n) \leqslant P_{\mathrm{b}}^{\mathrm{SBFR}_{-} 1}(n) \leqslant$ $P_{\mathrm{b}}^{\mathrm{SBFR} \_} 2(n)$, which shows that the proposed MBCFR scheme achieves the lowest blocking probability among these three frequency reuse schemes.

\section{Frequency segment sequence based MBCFR}

Since the whole resources are reused in each sector in the MBCFR, the number of interference beams for each user increases. In order to further decrease the ICI of the proposed MBCFR scheme, a frequency-segment-sequence based MBCFR (FSS-MBCFR) scheme is introduced in this section. Firstly, the cell-edge frequency subset and the cell-center frequency subset are both further divided into three orthogonal segments. Moreover, in order to control the inter-beam interference in a cooperative way, a cell-edge FSS is designed for each CCBS, while a cell-center FSS is designed for each beam. Each FSS defines its own base segment and allocation order. Finally, a RBs allocation rule is given based on the pre-designed FSSs, where the base segment is allocated with the highest priority, and the additional segments are added according to the allocation order of each FSS when the amount of traffic exceeds the capacity of the base segment.

\subsection{Frequency segment sequence design}

Every three neighboring cells are grouped into a cell cluster and respectively marked with Cell 1, Cell 2 and Cell 3 (see Fig. 4). Let $\mathrm{B}_{i} \mathrm{~S}_{j} \mathrm{C}_{n}$ denote the Beam $i$ of Sector $j$ in Cell $n$, where $i=\{1,2\}$ and $j, n=\{1,2,3\}$. Then, the CCBSs in the system are categorized into six types. The three types of CCBSs (CCBS-1, CCBS-2 and CCBS-3) composed of beams marked with Beam 1 are illustrated in Fig. 4. CCBS-1 is composed of $\mathrm{B}_{1} \mathrm{~S}_{1} \mathrm{C}_{1}, \mathrm{~B}_{1} \mathrm{~S}_{2} \mathrm{C}_{2}$ and $\mathrm{B}_{1} \mathrm{~S}_{3} \mathrm{C}_{3}$; CCBS-2 is composed of $\mathrm{B}_{1} \mathrm{~S}_{2} \mathrm{C}_{1}, \mathrm{~B}_{1} \mathrm{~S}_{3} \mathrm{C}_{2}$ and $\mathrm{B}_{1} \mathrm{~S}_{1} \mathrm{C}_{3}$; while CCBS-3 consists of $B_{1} S_{3} C_{1}, B_{1} S_{1} C_{2}$ and $B_{1} S_{2} C_{3}$. Similarly, three types of CCBSs composed of the beams marked with Beam 2 can be found. Note that the case of Beam 2 can be derived according to that of Beam 1. For simplicity, the principle of the FSS-MBCFR scheme is introduced based on the case of Beam 1.

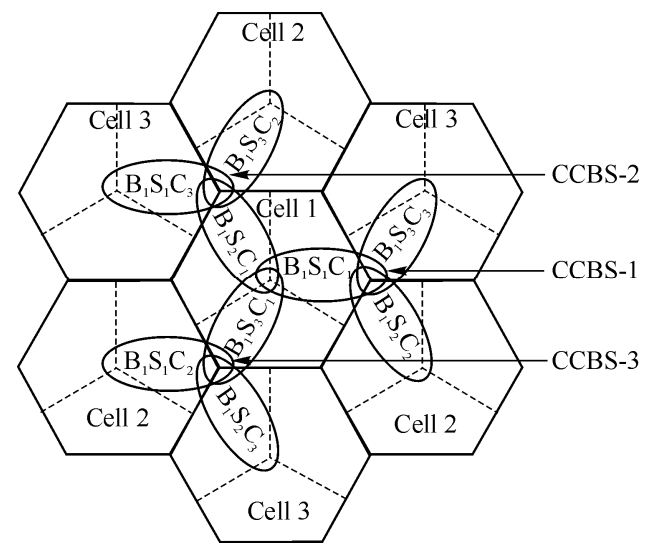

Fig. 4 Three types of CCBSs for beams marked with Beam 1

Firstly, the cell-edge frequency subset and the cell-center frequency subset of Beam 1 , denoted as $F_{1}^{\mathrm{E}}$ and $F_{1}^{\mathrm{C}}$, respectively, are further divided into three orthogonal segments, marked with $F_{1,1}^{\mathrm{E}}, F_{1,2}^{\mathrm{E}}, F_{1,3}^{\mathrm{E}}$ and $F_{1,1}^{\mathrm{C}}, F_{1,2}^{\mathrm{C}}, F_{1,3}^{\mathrm{C}}$, 
respectively. Then, in order to control the mutual interference among neighboring CCBSs, different cell-edge FSSs for CCBS-1, CCBS-2 and CCBS-3 are designed as follows:

1) Cell-edge FSS for CCBS-1: $F_{1,1}^{\mathrm{E}} \rightarrow F_{1,2}^{\mathrm{E}} \rightarrow F_{1,3}^{\mathrm{E}}$.

2) Cell-edge FSS for CCBS-2: $F_{1,2}^{\mathrm{E}} \rightarrow F_{1,3}^{\mathrm{E}} \rightarrow F_{1,1}^{\mathrm{E}}$.

3) Cell-edge FSS for CCBS-3: $F_{1,3}^{\mathrm{E}} \rightarrow F_{1,1}^{\mathrm{E}} \rightarrow F_{1,2}^{\mathrm{E}}$.

Different from CEUs, the major interference for each CCU comes from the two neighboring beams, since the CCU only communicates with its serving beam. For example, the major interfering beams for CCUs in $\mathrm{B}_{1} \mathrm{~S}_{1} \mathrm{C}_{1}$ are $\mathrm{B}_{1} \mathrm{~S}_{2} \mathrm{C}_{2}$ and $\mathrm{B}_{1} \mathrm{~S}_{3} \mathrm{C}_{3}$. Hence, in order to avoid the major inter-beam interference, these three neighboring beams should be assigned with different cell-center FSSs. Specifically, the cell-center FSSs for the beams marked with Beam 1 are designed as follows:

1) Cell-center FSS for Beam 1 of Sector $1: F_{1,1}^{\mathrm{C}} \rightarrow F_{1,2}^{\mathrm{C}} \rightarrow$ $F_{1,3}^{\mathrm{C}}$.

2) Cell-center FSS for Beam 1 of Sector $2: F_{1,2}^{\mathrm{C}} \rightarrow F_{1,3}^{\mathrm{C}} \rightarrow$ $F_{1,1}^{\mathrm{C}}$.

3) Cell-center FSS for Beam 1 of Sector 3: $F_{1,3}^{\mathrm{C}} \rightarrow F_{1,1}^{\mathrm{C}} \rightarrow$ $F_{1,2}^{\mathrm{C}}$.

We define the first segment in each FSS as the base segment. According to the pre-designed FSSs, neighboring CCBSs are assigned with different cell-edge based segment so that the ICI for CEUs can be effectively decreased when the traffic load is light. For CCUs, note that neighboring beams have different cell-center base segment, so the ICI for CCUs can also be avoided.

\subsection{RBs allocation rule}

Based on the pre-designed FSSs, the RBs allocation rule for CEUs in each CCBS is given as follows:

Step 1 If the number of CEUs in the CCBS does not exceed the capacity of its base segment, the subcarriers in the corresponding base segment are randomly allocated to the demanding users.

Step 2 If the number of CEUs exceeds the base segment capacity, additional segments are added according to the segment allocation sequence of the CCBS. The RBs within the corresponding base segment are allocated with the highest priority, and then the remainder of requested RBs is randomly allocated within the new added segment.

Step 3 This process continues until the three segments of the CCBS are exhausted.
The RBs allocation rule for CCUs in each beam can be derived according to the one designed for CEUs.

According to the proposed RBs allocation rule, the number of interference beams due to co-channel interference can be effectively decreased for both CEUs and CCUs when the traffic load is less than the base capacity. Hence, the major ICI can be effectively avoided when the traffic load is light. When the traffic load exceeds the base capacity, the ICI is averaged over the newly added segments by random RBs allocation.

\section{Performance analysis}

In this section, system level simulations are performed to evaluate the performance of the proposed MBCFR and FSS-MBCFR schemes. The frequency reuse schemes in Refs. [10-11] are taken as reference schemes, named as SBFR-1 and SBFR-2, respectively. As shown in Fig. 3, in the two reference schemes, each sector is assigned with one third of the system available RBs. Besides, in the SBFR-1 scheme, the RBs for each beam are randomly assigned to the different users irrespective of their category (CEU or CCU). While in the SBFR-2, the MBCFR and the FSS-MBCFR schemes, the case of using different subcarriers for CEU and CCU is considered, and the long term gain threshold is predefined as $-100 \mathrm{~dB}$.

We focus on a downlink OFDMA switched-beam system. A given number of users are uniformly dropped within each cell. For all the four schemes, each sector is covered by two beams and configured with four transmitting antennas, while one receiving antenna is considered for each user. Assume that no power control is applied, and that all the available RBs are transmitted with equivalent power. According to the proportion of the number of CCUs and CEUs, the number of RBs in different frequency subsets is predefined as follows: $\left|F_{1}\right|=\left|F_{2}\right|=25,\left|F_{1}^{\mathrm{E}}\right|=\left|F_{2}^{\mathrm{E}}\right|=6,\left|F_{1}^{\mathrm{C}}\right|=\left|F_{2}^{\mathrm{C}}\right|=19$.

The blocking probability, cell-edge average throughput and cell-average throughput are evaluated for performance analysis. The cell-edge performance is evaluated by averaging the performances of the $5 \%$ weakest users, while the cell-average performance is obtained by averaging the throughput of the entire users within the cell. Table 1 lists the main simulation parameters, based on Ref. [2].

Fig. 5 shows the blocking probability as a function of the number of users per cell. The blocking probability of different schemes is respectively calculated according to Eqs. (11)-(13). It can be seen that the proposed MBCFR and FSS-MBCFR 
schemes achieve almost the same performance in terms of blocking probability, since the number of available RBs for each beam in these two schemes is the same according to the pre-designed frequency reuse rule. We can also observe that the proposed MBCFR and FSS-MBCFR schemes outperform the two reference schemes. As analyzed in Sect. 3, this improvement in the proposed schemes results from the full frequency reuse in each sector. According to the frequency reuse rule, the whole frequency resources can be reused in all sectors in the MBCFR and FSS-MBCFR schemes. Hence, the number of available RBs for each sector in the proposed MBCFR and FSS-MBCFR schemes is twice more than the two reference schemes.

Table 1 Simulation parameters

\begin{tabular}{lc}
\hline \multicolumn{1}{c}{ Parameter } & Value \\
\hline Bandwidth & $10 \mathrm{MHz}$ \\
Subcarrier spacing & $15 \mathrm{kHz}$ \\
Number of RBs & 50 \\
Number of cells & 19 \\
Cell radius & $500 \mathrm{~m}$ \\
Maximum power in BS & $46 \mathrm{dBm}$ \\
Distance-dependent path loss & $L=(128.1+37.61 \mathrm{~g} \mathrm{~d}) / \mathrm{dB}, \mathrm{d} / \mathrm{km}$ \\
Shadowing factor variance & $8 \mathrm{~dB}$ \\
Shadowing correlation distance & $50 \mathrm{~m}$ \\
Target BER & $10^{-6}$ \\
\hline
\end{tabular}

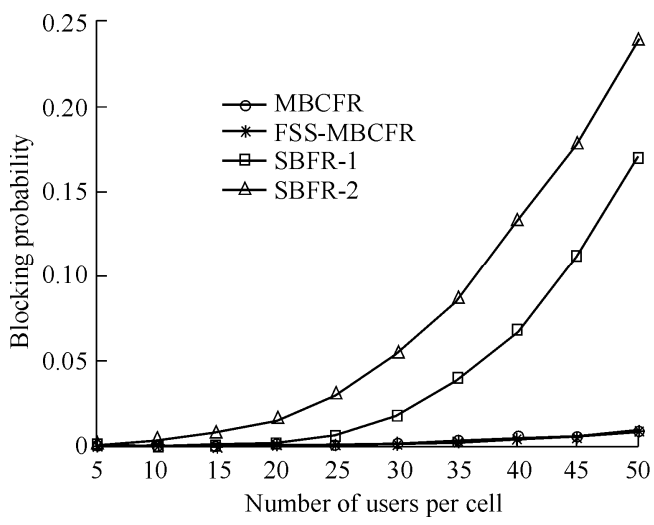

Fig. 5 Blocking probability of the four schemes as a function of the number of users per cell

Fig. 6 shows the cell-edge average throughput per user of the four frequency reuse schemes. It can be seen that the cell-edge average throughput of the MBCFR scheme is slightly higher than the SBFR-1 scheme, while lower than the SBFR-2 scheme. Since the whole resources are reused in each sector in the MBCFR, the number of interference beams for each user increases compared with the two reference schemes. Hence, the multi-beam joint transmission gain is compromised by the increased ICI.

However, the FSS-MBCFR scheme significantly improves the cell-edge performance compared with the two reference schemes. The reason is that the major ICI from the interfering beams can be effectively avoided by the predesigned frequency segment sequences. Hence, the multi-beam joint transmission gain can be significantly achieved. As shown in Fig. 6, when the traffic load is light, e.g. 10 users per cell, the cell-edge average throughput is improved by $226 \%$ more than the SBFR-1 scheme, and $166 \%$ more than the SBFR-2 scheme. While when the number of users per cell is large, e.g. more than 40, the improvement gain decreases. However, the payoff for the cell-edge performance of the two reference schemes is a huge increase in the blocking probability, which can be observed from Fig. 5 .

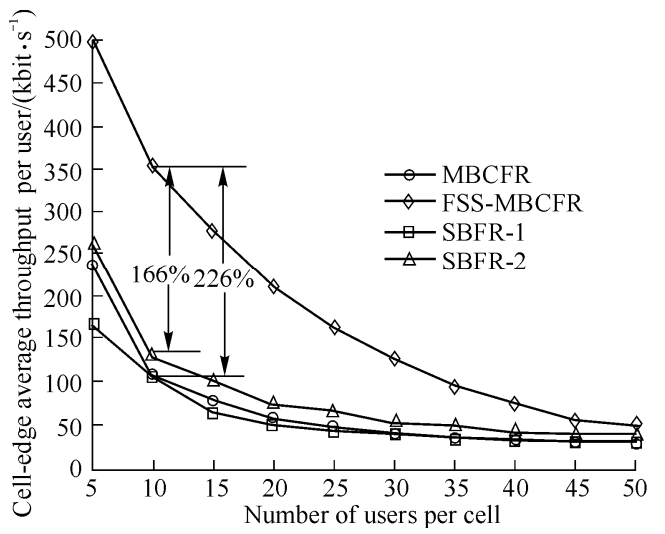

Fig. 6 Cell-edge average throughput per user as a function of the number of users per cell

Fig. 7 shows the cell-average throughput of the four schemes. From the graph, we can see that the cell-average throughput of the MBCFR scheme outperforms that of the two reference schemes, $4 \%$ to $15 \%$ more than the SBFR-1 scheme and $6 \%$ to $19 \%$ more than the SBFR-2 scheme. The FSS-MBCFR scheme slightly outperforms the MBCFR scheme, since the ICI is further decreased by the predefined frequency segment sequences.

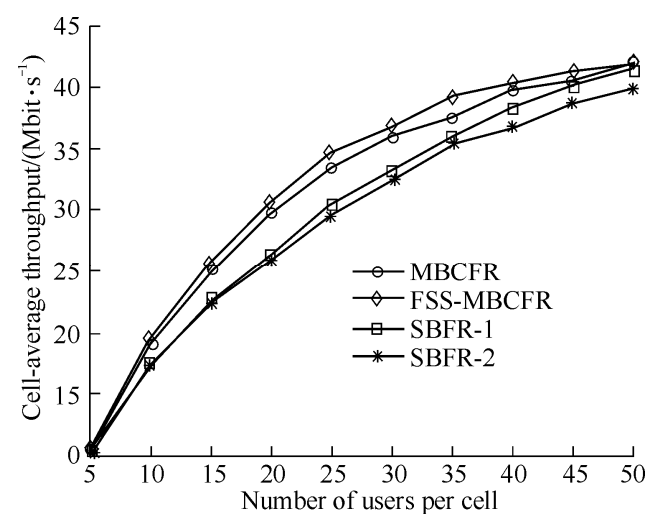

Fig. 7 Cell-average throughput as a function of the number of users per cell 


\section{Conclusions}

In this paper, a novel frequency reuse scheme is proposed for an OFDMA switched-beam system to support CoMP joint transmission and further improve the cell-edge performance. Firstly, a multi-beam cooperative frequency reuse scheme is designed, which assigns neighboring beams in each sector with orthogonal frequency sets, and allows the beams belonging to the same CoMP cooperating beam-set to reuse the same frequency resources in cell-edge areas so as to support multi-beam joint transmission. Besides, the blocking probability per sector is calculated, which shows that the proposed scheme outperforms the two reference frequency reuse schemes in Refs. [10-11] with the lowest blocking probability. Then, a frequency segment sequence based multi-beam cooperative frequency reuse scheme, named as FSS-MBCFR, is introduced to further decrease the inter-beam interference. The FSS-MBCFR scheme designs a cell-center frequency segment allocation sequence for each CoMP cooperating beam-set and a cell-center frequency segment allocation sequence for each beam. Each sequence defines its own base segment and allocation order. The base segment is allocated with the highest priority, and the additional segments are added according the allocation order of each sequence when the amount of traffic exceeds the capacity of the base segment. Hence, the major interference among neighboring beams can be effectively avoided in a cooperative way. System level simulation results demonstrate that the proposed FSS-MBCFR scheme results in higher average throughput in both cell-edge and cell-average points of view with lower blocking probability.

\section{Acknowledgements}

This work was supported by the Sino-Swedish Project
(2008DFA12110), the Key Project of BMSTC (D08080100620802), the National Natural Science Foundation of China (60872048), and the National Science and Technology Special Project 'Group Cell' ( 2009ZX03003-011).

\section{References}

1. Physical layer aspects for evolved UTRA (Release 7). 3GPP TR 25.814 v7.1.0. 2006

2. Further advancements for E-UTRA physical layer aspects (Release 9). 3GPP TR 36.814 v1.5.0. 2009

3. Boudreau G, Panicker J, Guo N, et al. Interference coordination and cancellation for 4G networks. IEEE Communications Magazine, 2009, 47(4): 74-81

4. A discussion on some technology components for LTE-advanced. 3GPP R1-082024. 2008

5. Karakayali M K, Foschini G J, Valenzuela R A. Network coordination for spectrally efficient communications in cellular systems. IEEE Wireless Communications Magazine, 2006, 13(4): 56-61

6. Soft frequency reuse scheme for UTRAN LTE. 3GPP R1-050507.2005

7. Tomcik J. MBFDD and MBTDD wideband mode: technology overview. IEEE 802.20-05/68r1. 2006

8. Elayoubi S E, Haddada O B, Fourestie B. Performance evaluation of frequency planning schemes in OFDMA-based networks. IEEE Transactions on Wireless Communications, 2008, 7(5): 1623-1633

9. Giuliano R, Monti C. WiMAX fractional frequency reuse for rural environments. IEEE Transactions on Wireless Communications, 2008, 15(3): 60-65

10. Nasri R, Kammoun A, Stephenne A, et al. System-level evaluation of a downlink OFDM switched-beam system with Kalman-based joint channel estimation and beam selection. Proceedings of the 24th Biennial Symposium on Communications, Jun 24-26, 2008, Kingston, Canada. Piscatawaw, NJ, USA: IEEE, 2008: 31-34

11. Nasri R, Kammoun A, Stephenne A, et al. System-level evaluation of a downlink OFDM Kalman-based switched-beam system with subcarrier allocation strategies. Proceedings of the 68th Vehicular Technology Conference (VTC-Fall'08), Sep 21-24, 2008, Calgary, Canada. Piscataway, NJ, USA: IEEE, 2008: 5p

12. Tse D,Viswanath P. Fundamentals of wireless communications. London, UK: Cambridge University Press, 2006

13. Goldsmith A J, Chua S G. Variable-rate variable-power MQAM for fading channels. IEEE Transactions on Communications, 1997, 45(10): 1218-1230 